integrated numerically to obtain the value $y_{1}(1)=y_{2}(0)$. The three equations $(2.1)(0),(2.1)(1),(2.1)(2)$ are integrated numerically to obtain the initial value $y_{2}(1)=y_{3}(0)$, etc. Since $y_{j}(x)=y(x+j), j=0,1, \cdots, N$, we obtain in this way the solution $y(x)$ in $0 \leqq x \leqq N+1$.

3. Numerical Results of Digital Computer Experiments. Using an IBM 7090 Fortran program with integration subroutines INT and INTM from the IBM Share Library D2 RWFINT and a fixed grid size $H$, our results agreed to eight significant figures up to $N=5$ for $H=2^{-7}$ and $H=2^{-8}$. As $N$ increased, the agreement got poorer and at $x=20$ there was agreement to only three significant figures with the initial value $y_{1}(0)=1$.

4. Stability. Examining the related system of differential equations, we note that the characteristic values of the matrix of coefficients are all zero. Consequently, we are on the borderline of stability, and progressive loss of accuracy is to be expected as $N$ increases. Using finer grids and more precise methods, we could, of course, decrease the rate of loss of accuracy. In an earlier computation of the solution of differential-difference equations [3] this effect was not present, and more accurate results were obtained.

5. Tables. In this section we present two tables of values. The first presents values of $y(x)$ at intervals of $\frac{1}{16}$ accurate to six or more significant figures. The second presents subsequent values to the degree of accuracy we possess. Observe that as $x$ increases, the number of significant figures decreases.

The RAND Corporation

Santa Monica, California

1. N. G. DEBRUIJN, "On the number of positive integers $<x$ and free of prime factors $>y$," Nederl. Akad. Wetensch. Proc. Ser. A, v. LIV, n. 1, 1951, p. 1-12.

2. R. Bellman, "On the computational solution of differential-difference equations," J. Math. Anal. Appl., v. 2, 1961, p. 108-110.

3. R. Bellman, \& B. Kotkin, On the Computational Solution of a Class of Nonlinear Differential-Difference Equations, The RAND Corporation, Paper P-2233, February 1961.

\title{
The Coefficients of the Lemniscate Function
}

\section{By L. Carlitz}

Let $\wp(u)$ denote the special Weierstrass $\wp$-function that satisfies the differential equation

$$
\wp^{\prime 2}(u)=4 \wp^{3}(u)-4 \wp(u)
$$

Hurwitz [4] put

$$
\wp(u)=\frac{1}{u^{2}}+\frac{2^{4} E_{1}}{4} \frac{u^{2}}{2 !}+\frac{2^{8} E_{2}}{8} \frac{u^{6}}{6 !}+\cdots+\frac{2^{4 n} E_{n}}{4 n} \frac{u^{4 n-2}}{4 n-2}+\cdots .
$$

Received March 26, 1962. The research reported in this paper was supported in part by the National Science Foundation. 
He showed that the $E_{n}$ satisfy the recurrence

$$
\text { (1) } \quad E_{n}=\frac{3}{(2 n-3)\left(16 n^{2}-1\right)} \sum_{k=1}^{n-1}(4 k-1)(4 n-4 k-1)\left(\begin{array}{l}
4 n \\
4 k
\end{array}\right) E_{k} E_{n-k}
$$

from which it follows that the $E_{n}$ are positive rational numbers.

Hurwitz proved also that

$$
\sum^{\prime} \frac{1}{(r+s i)^{4 n}}=\frac{(2 \omega)^{4 n}}{(4 n) !} E_{n}
$$

where the summation is over all complex integers $r+s i$ except 0 and

$$
\omega=2 \int_{0}^{1} \frac{d x}{\sqrt{1-x^{4}}} .
$$

The most interesting property proved by Hurwitz for the $E_{n}$ is the following analog of the Staudt-Clausen theorem. Let $p$ be a prime of the form $4 k+1$ so that $p=a^{2}+b^{2} ;$ we take $a$ odd and such that

Then

$$
a \equiv b+1 \quad(\bmod 4) .
$$

$$
E_{n}=G_{n}+\frac{1}{2}+\sum \frac{(2 a)^{4 n /(p-1)}}{p}
$$

where the summation is over all primes $p$ of the form $4 k+1$ such that $p-1 \mid 4 n ; G_{n}$ is an integer.

Hurwitz computed the first twelve values of $E_{n}$ both in the form (2) and also in the form

$$
E_{n}=N_{n} / D_{n} \quad\left(N_{n}, D_{n}\right)=1,
$$

where $N_{n}$ denotes the numerator and $D_{n}$ the denominator of $E_{n}$ in reduced form.

The present writer [2] has discussed arithmetic properties of the coefficients of singular elliptic functions. In particular, he showed that if $p$ is a prime of the form $4 k+3$ then

$$
E_{m} \equiv 0 \quad\left(\bmod p^{r+s}\right)
$$

when $p^{8} \mid m, p^{2}-1 \nmid 4 m$ and $4 m>p r+1$, while

$$
E_{m} \equiv 0 \quad\left(\bmod p^{r-1}\right)
$$

when $p^{8}\left|m, p^{2}-1\right| 4 m$ and $4 m>p r+1$. In some cases, but not all, (4) and (5) predict the correct power of the primes $p$ dividing $E_{m}$. However, the formulas give no information about the occurrence in the numerator of $E_{n}$ about primes of the form $4 k+1$. An interesting question is whether infinitely many primes

$$
p \equiv 1 \quad(\bmod 4)
$$

occur in the numerators of $E_{m}$.

In a later paper [3] the writer obtained congruences $\left(\bmod 2^{r}\right)$ for the $E_{n}$ as well as for the coefficients of certain related functions. In particular, he showed that

$$
\sum_{s=0}^{r}(-1)^{s}\left(\begin{array}{l}
r \\
s
\end{array}\right) 2 E_{n+8 z} \equiv 0 \quad\left(\bmod 2^{r e}\right),
$$

provided $2^{e-1} \mid z, \quad r \geqq 0$ and $n>r e$. 
In order to get additional information about the numerators $N_{n}$ of $E_{n}$, it was thought desirable to compute some additional values of $N_{n}$. The following values were computed by $\mathrm{R}$. Carlitz in the Duke University Computing Laboratory, making use of the recurrence (1). We remark that the first twelve values are in agreement with the results given by Hurwitz. We write $N_{n}$ for the numerator of $E_{n}$ as defined in (3).

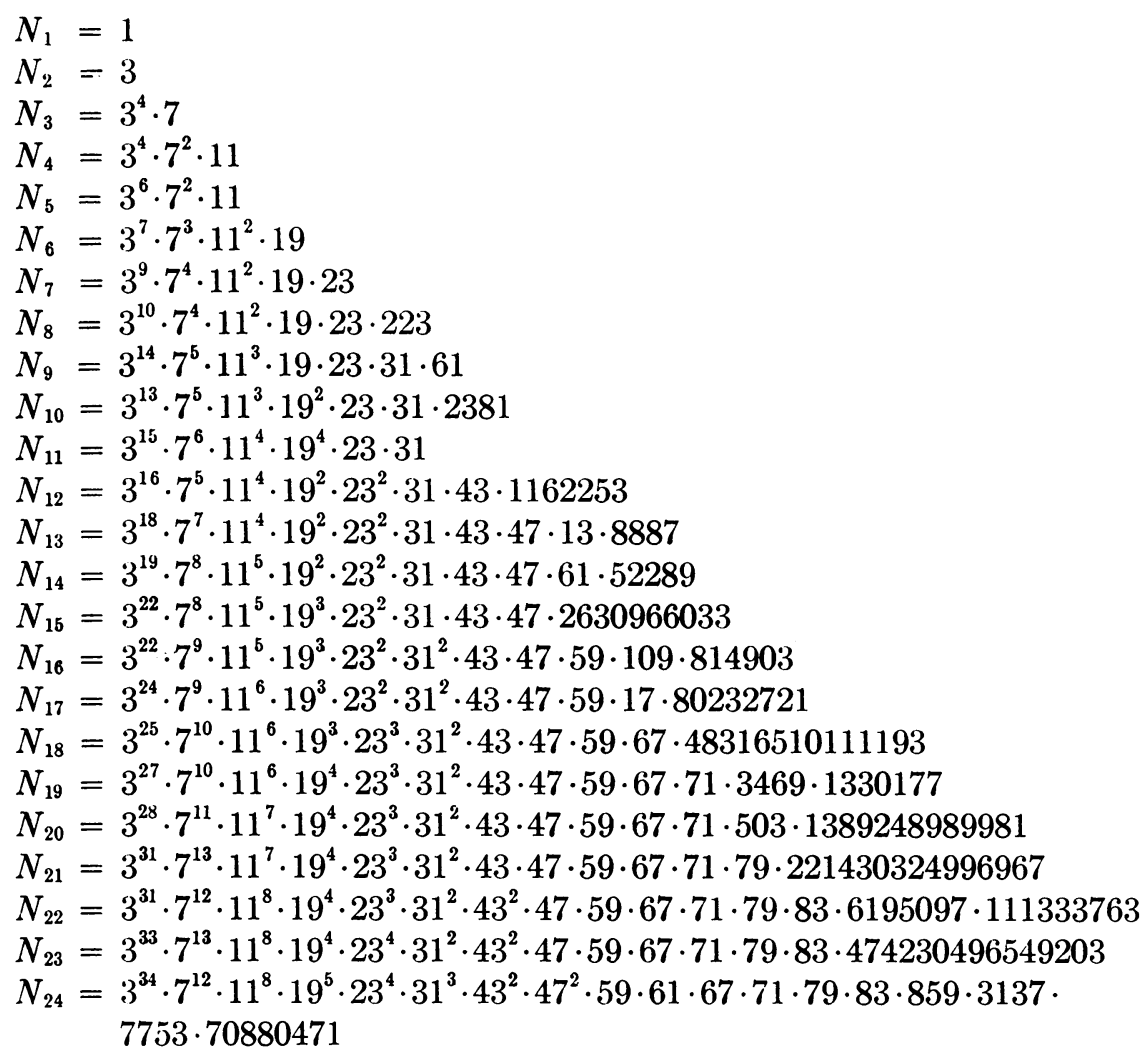

In every case the $N_{n}$ has been factored completely.

It will be noted that $N_{13} \equiv 0(\bmod 13)$ and $N_{17} \equiv 0(\bmod 17)$. This suggests the following

TheоRem. If $p$ is a prime greater than 5, we have

$$
N_{p} \equiv 0 \quad(\bmod p) .
$$

More generally if

$$
p^{r} \mid m, \quad p-1 \nmid 4 m, \quad(-1)^{m} 2^{2 m-1} \not \equiv 1 \quad(\bmod p)
$$

then

$$
N_{m} \equiv 0 \quad\left(\bmod p^{r}\right)
$$

Proof. If we put

$$
\frac{u}{\phi(u)}=\sum_{m=0}^{\infty} \beta_{4 m} \frac{x^{4 m}}{(4 m) !} \quad\left(\phi(u)=(\wp(u))^{-1 / 2}\right)
$$


then, as Hurwitz showed,

$$
\beta_{4 m}=(1+i)^{4 m}\left\{(1+i)^{4 m}-2\right\} E_{m} .
$$

The writer [1, Theorem 9] has proved that if $p^{r} \mid m, p-1 \nmid 4 m$, then

Thus (10) yields

$$
\beta_{4 m} \equiv 0 \quad\left(\bmod p^{r}\right) .
$$

$$
\left\{(1+i)^{4 m}-2\right\} E_{m} \equiv 0 \quad\left(\bmod p^{r}\right) .
$$

Since $p-1 \nmid 4 m$, the denominator of $E_{m}$ is not divisible by $p$, so that (11) implies

$$
\left\{(1+i)^{4 m}-2\right\} N_{m} \equiv 0 \quad\left(\bmod p^{r}\right) .
$$

In the next place, since

it is evident that

$$
(1+i)^{4 m}=(-4)^{m}
$$

$$
(1+i)^{2 m}-2 \equiv 0 \quad(\bmod p)
$$

if and only if

$$
(-1)^{m} 2^{2 m-1} \equiv 1 \quad(\bmod p) .
$$

If, therefore, (13) is not satisfied, it is clear from (12) that

$$
N_{m} \equiv 0 \quad\left(\bmod p^{r}\right) \text {. }
$$

Finally we note that if $p=m$, then (13) is not satisfied and (7) follows at once.

Duke University

Durham, North Carolina

1. L. CarLITZ, "Congruences connected with the power series expansions of the Jacobi elliptic functions," Duke Math. J., v. 20, 1953, p. 1-12.

2. L. CARLitz, "The coefficients of singular elliptic functions," Math. Ann., v. 127, 1954, p. $162-169$.

3. L. CARLITz, "Some arithmetic properties of the lemniscate coefficients," Math. Nachr., v. 22,1960, p. $237-249$.

4. A. Hurwitz, "Entwickelungscoeffizienten der lemniscatischen Funktionen," Math. Ann., v. 51, 1899, p. 196-226 (Mathematische Werke, Basel, 1953, v. 2, p. 342-373).

\section{All Factors $q<10^{8}$ in All Mersenne Numbers $2^{p}-1, p$ Prime $<10^{4}$}

\section{By H. Riesel}

During the year 1960 the author made additional investigations respecting factors of Mersenne numbers $M_{p}=2^{p}-1$, where $p$ is a prime. The author has earlier examined the least factor $q$ of $M_{p}$, for $p<10^{4}$, if $q<10 \cdot 2^{20} \approx 10^{7}$. This inquiry was secondary to an effort to ascertain Mersenne primes [1].

The present examination, which resulted in 255 new factors of the numbers $M_{p}$, was done in the following manner: All primes $s<10^{8}$, for which $s \equiv 1(\bmod 2 p)$ and $s \equiv 1 \pm(\bmod 8)$ were tested as factors of $M_{p}$ for $1200<p<10000$. The range $p<1200$ has been examined by Brillhart and Johnson [2].

Received February 10, 1961. 